Patients who had emergent angiography after presenting with clinical stroke during the post-surgical period were included. Type of surgery, clinical characteristics, imaging studies, treatment variables, and discharge modified Rankin scores were collected.

Results 15 patients had emergent angiography in the post-surgical period. The mean age is 68.87 years. $87 \%(13 / 15)$ had cardiovascular surgery, $8(62 \%)$ of which were valvular repairs. $57 \%(8 / 14)$ presented in the immediate post-surgical period. The mean NIHSS was 18. Mean symptom duration from time last known well was 256 minutes. 10/11 (91\%) had perfusion deficits on CTP.

$60 \%(9 / 15)$ had proximal vessel occlusion while $33 \%$ $(5 / 15)$ had distal occlusions, and 1 had no occlusion. Of the 9 patients with proximal vessel occlusions, 7 underwent thrombectomy with the Solitaire device achieving a 57\% (4/7) TICI $2 \mathrm{~b} / 3$ recanalization rate. The mean mRS for all patients in the series is 3.79. Patients with TICI $2 \mathrm{~b} / 3$ recanalization did significantly better at discharge than those who did not, mean mRS of 1.67 vs. $5, \mathrm{p}=0.019$.

Conclusion In our experience, emergent angiography among post-surgical stroke patients showed proximal vessel occlusion in more than half of patients, making them potential candidates for mechanical thrombectomy. Those who achieved TICI $2 \mathrm{~b} / 3$ recanalization performed clinically better than those who did not. This may indicate the potential benefit of mechanical thrombectomy among post-surgical stroke patients. The overall lower recanalization rates in this cohort may reflect atypical clot characteristics encountered in post-surgical patients and would be an interesting avenue for further investigation.

Disclosures M. Litao: None. H. Bhamra: None. K. DeSousa: None. E. Raz: None. E. Nossek: None. A. Favate: None. M. Shapiro: 2; C; Covidien. T. Becske: 2; C; Covidien. P. Nelson: 2; C; Covidien.

\section{E-028 MANAGEMENT OF TONGUE VENOUS AND LYMPHATIC MALFORMATIONS}

W Yakes. Vascular Malformation Center, Englewood, CO

\subsection{6/neurintsurg-2016-012589.100}

Purpose To determine the efficacy of ethanol embolization in management of tongue venous and lymphatic malformations. Materials and methods Thirty-nine patients (22 females, 17 males; mean age: 38 years) presented with tongue low-flow malformations. Forty-seven patients had undergone 61 failed previous procedures (embo, laser, surgery, steroid injection, alpha-interpheron, radiation). All patients had baseline arteriograms and MRs. All patients underwent direct puncture ethanol endovascular therapy.

Results Of 39 patients with venous and lymphatic malformations, 32 patients had dramatic reduction and 7 patients' therapy is on-going with concurrent reductions (mean f/up: 60 months). One patient with AVM required additional surgery and 1 patient with mixed veno-lymphatic malformation required surgical debulking of excess tissues. Minor complications such as tongue blisters ( 9 instances) healed spontaneously; 3 tongue focal necrosis injuries healed spontaneously; 3 infections responded to antibiotic treatment; 1 focal tongue hemi numbness resolved.
Conclusion Ethanol embolotherapy is a primary and consistent form of therapy to eradicate low-flow vascular malformations of the tongue permanently at long-term follow-up. Rarely is concurrent surgery required. Ethanol sclerotherapy is a curative treatment in which recurrences do not occur and permanent ablations are the rule. Complications that occur are minor and rare.

Disclosures W. Yakes: None.

\section{E-029 MECHANICAL THROMBECTOMY IN PEDIATRIC ACUTE ISCHEMIC STROKE: CLINICAL OUTCOMES AND LITERATURE REVIEW}

${ }^{1} \mathrm{~T}$ Madaelil, ${ }^{1} \mathrm{~A}$ Kansagra, ${ }^{2} \mathrm{C}$ Derdeyn, ${ }^{1} \mathrm{D}$ Cross, ${ }^{1} \mathrm{C}$ Moran. ${ }^{1}$ Neuroradiology, Mallinckrodt Institute of Radiology, Saint Louis, MO; ${ }^{2}$ Neuroradiology, Neurology, Neurosurgery, University of lowa Hospitals and Clinics, lowa City, IA

\subsection{6/neurintsurg-2016-012589.101}

There is limited data on outcomes of mechanical thrombectomy for pediatric stroke using modern devices. In this study, we report two cases of pediatric acute ischemic stroke treated with mechanical thrombectomy, both with good angiographic result (TICI 3) and clinical outcome (no neurological deficits at 90 days). In addition, we conducted a literature review of all previously reported cases describing the use of modern thrombectomy devices. Including our two cases, the aggregate rate of partial or complete vessel recanalization was $100 \%$ $(22 / 22)$, and the aggregate rate of favorable clinical outcome was 91\% (20/22). This preliminary evidence suggests that mechanical thrombectomy with modern devices may be a safe and effective treatment option in pediatric patients with acute ischemic stroke.

Disclosures T. Madaelil: None. A. Kansagra: None. C. Derdeyn: None. D. Cross: None. C. Moran: 2; C; Medtronic Neurovascular.

\section{E-030 TRANSVENOUS MICROGUIDEWIRE LOOPING TECHNIQUE FOR BREACH OF IPSILATERAL INFERIOR PETROSAL SINUS OCCLUSIONS EN ROUTE TO CAVERNOUS SINUS DURAL ARTERIOVENOUS FISTULAS}

Y Cho, H Kang, M Han. Radiology, Seoul National University Hospital, Seoul, Republic of Korea

10.1136/neurintsurg-2016-012589.102

Purpose Transarterial access to dural arteriovenous fistulas (dAVFs) has been popularized by device improvements and novel embolic materials. However, this approach is limited in the cavernous sinus (CS) due to related complications and low cure rates. Although a transvenous approach, via ipsilateral inferior petrosal sinus (IPS), may be more suitable for CSdAVFs, microcatheter delivery is occasionally impeded by ipsilateral IPS occlusion. Described herein is a novel microguidewire looping method to breach such occlusions, thus enabling access to CS lesions.

Methods A microcatheter is initially advanced into IPS orifice, and a microguidewire is passed into occluded IPS. Looping is easily achieved through the resistance met. With greater support of the guiding catheter, the microguidewire (still looped) is then advanced into CS. When nearing CS, the 